\title{
Rationalizability and minimal complexity in dynamic games
}

Citation for published version (APA):

Perea ý Monsuwé, A. (2003). Rationalizability and minimal complexity in dynamic games. METEOR, Maastricht University School of Business and Economics. METEOR Research Memorandum No. 030 https://doi.org/10.26481/umamet.2003030

Document status and date:

Published: 01/01/2003

DOI:

10.26481/umamet.2003030

Document Version:

Publisher's PDF, also known as Version of record

\section{Please check the document version of this publication:}

- A submitted manuscript is the version of the article upon submission and before peer-review. There can be important differences between the submitted version and the official published version of record.

People interested in the research are advised to contact the author for the final version of the publication, or visit the DOI to the publisher's website.

- The final author version and the galley proof are versions of the publication after peer review.

- The final published version features the final layout of the paper including the volume, issue and page numbers.

Link to publication

\footnotetext{
General rights rights.

- You may freely distribute the URL identifying the publication in the public portal. please follow below link for the End User Agreement:

www.umlib.nl/taverne-license

Take down policy

If you believe that this document breaches copyright please contact us at:

repository@maastrichtuniversity.nl

providing details and we will investigate your claim.
}

Copyright and moral rights for the publications made accessible in the public portal are retained by the authors and/or other copyright owners and it is a condition of accessing publications that users recognise and abide by the legal requirements associated with these

- Users may download and print one copy of any publication from the public portal for the purpose of private study or research.

- You may not further distribute the material or use it for any profit-making activity or commercial gain

If the publication is distributed under the terms of Article $25 \mathrm{fa}$ of the Dutch Copyright Act, indicated by the "Taverne" license above, 


\title{
Rationalizability and Minimal Complexity in Dynamic Games
}

\author{
Andrés Perea* \\ Maastricht University
}

June 2003

\begin{abstract}
This paper presents a formal epistemic framework for dynamic games in which players, during the course of the game, may revise their beliefs about the opponents' utility functions. We impose three key conditions upon the players' beliefs: (a) throughout the game, every move by the opponent should be interpreted as being part of a rational strategy, (b) the belief about the opponents' relative ranking of two strategies should not be revised unless one is certain that the opponent has decided not to choose one of these strategies, and (c) the players' initial beliefs about the opponents' utility functions should agree on a given profile $u$ of utility functions. Types that, throughout the game, respect common belief about these three events, are called persistently rationalizable for the profile $u$ of utility functions. It is shown that persistent rationalizability implies the backward induction procedure in generic games with perfect information. We next focus on persistently rationalizable types for $u$ that hold a theory about the opponents of "minimal complexity", resulting in the concept of minimal rationalizability. For two-player simultaneous move games, minimal rationalizability is equivalent to the concept of Nash equilibrium strategy. In every outside option game, as defined by van Damme (1989), minimal rationalizability uniquely selects the forward induction outcome.

Keywords: Rationalizability, belief revision, dynamic games, backward induction, forward induction.

JEL Classification: C72
\end{abstract}

\section{Introduction}

In the epistemic approach to noncooperative games every player is modeled as a decision maker under uncertainty, endowed with a preference ordering on the possible strategy choices. Under the assumption that each player is of the expected utility type, such preference orderings may be represented by a utility function over the possible consequences and a subjective probability distribution, or belief, over the uncertain parameters in the game. Most epistemic models that have been proposed in the literature assume that the players face no uncertainty about

\footnotetext{
*Department of Quantitative Economics, Maastricht University, P.O. Box 616, 6200 MD Maastricht, The Netherlands. E-mail: a.perea@ke.unimaas.nl
} 
the opponents' utility functions (some papers that explicitly allow for uncertainty about the opponents' utility functions will be discussed below). This property is usually modeled by the presence of an exogenously given profile of utility functions and the implicit requirement that, whatever happens in the game, these utility functions are never to be questioned. The uncertainty faced by a player at a given instance of the game will then consist of the opponents' strategy choices, the opponents' beliefs about the other players' strategy choices, the opponents' beliefs about the other players' beliefs about the other players' strategy choices, and so forth.

Within a given epistemic model for games, the problem of how to model rational behavior cannot be reduced to one-person decision theory since a player should not only choose rationally given his beliefs, but these beliefs should also be based upon the conjecture that his opponents choose rationally as well. Also should a player realize that each of his opponents will hold beliefs that are based upon the conjecture that the other players act rationally, and so on. This intuitive argument may be formalized by the notion of common belief of rationality, a concept that plays a central role in theories of rationality such as rationalizability (Bernheim (1984) and Pearce (1984)), Nash equilibrium and all refinements thereof. Indeed, Tan and Werlang (1988) have shown that, within a formal epistemic model, the strategies that may be chosen rationally when there is common belief of rationality coincide exactly with the set of rationalizable strategies.

A fundamental problem arises, however, if the notion of common belief of rationality is to be applied to dynamic games, and no uncertainty about the utility functions is allowed. The difficulty is that there may be information sets in the game that cannot be reached if players were to act in accordance with common belief of rationality. Reny (1992a, 1993) has shown that for the class of perfect information games, this phenomenon occus on a rather structural basis. A natural question which then arises is: how should a player revise his beliefs about the opponents' strategy choices and the opponents' beliefs if an information set is reached that contradicts common belief of rationality? At this stage, the player should conclude that there is at least one opponent who (a) did not act rationally given his beliefs, or (b) bases his beliefs upon the conjecture that some other player does not act rationally given his belief, or (c) believes that some other player believes that some other player acts irrationally, and so on. A concept of rationality should specify which of the above scenarios is to be viewed as "most plausible", thus imposing a restriction on how beliefs are to be revised at such "problematic" information sets.

In the literature, several rationalizability concepts for dynamic games have been proposed that hold different views on how to revise beliefs when common belief of rationality has been contradicted by the play of the game. The concept of common certainty of rationality at the beginning of the game (Ben-Porath (1997)) and its extension to general dynamic games, to which we shall refer as weak sequential rationalizability, require common belief at the beginning of the game about the event that players choose rationally at each of their information sets, but impose no restriction upon the players' belief revisions at information sets where the player's initial belief about the opponents has been contradicted. In particular, if common belief of rationality has been contradicted at a given information set, the corresponding player may from now on believe that one or more opponents chooses suboptimally.

Backward induction, and backward induction based rationalizability concepts such as sequential and quasi-perfect rationalizability (Asheim and Perea (2002)), state that a player, at an 
information set where common belief of rationality has been contradicted, should conclude that the event of reaching this information set is due to a suboptimal move by one of his opponents, but should maintain his belief in common belief of rationality for the remainder of the game.

The concept of extensive form rationalizability (Pearce (1984) and Battigalli (1997)) holds yet another viewpoint by requiring that a player, at an information set contradicting common belief of rationality, should not conclude immediately that an opponent has chosen suboptimally, but should rather seek for the "highest possible degree of interactive belief of rationality" that is compatible with the event of reaching this information set. From then on, the player should base his beliefs about the opponents upon this degree of interactive belief of rationality until some further information set is reached that contradicts this degree. At this occasion, the player should again search for the highest possible degree of interactive belief of rationality that explains the event of reaching this information set, and so on.

In this paper we choose an alternative approach by allowing the players to revise their beliefs about the opponents' utility functions during the game, while insisting on common belief of rationality at every possible instance in the game (see Perea (2002) for a similar approach within an equilibrium framework). Accordingly, we develop an epistemic model in which every player, at each of his information sets, has uncertainty about the opponents' strategy choices, the opponents' utility functions, the opponents' first-order beliefs about the other players' strategy choices, the opponents' first-order beliefs about the other players' utility functions, the opponents' second-order beliefs about the other players' first-order beliefs, etcetera. This leads, for every player at each of his information sets, to an infinite hierarchy of succesively richer uncertainty spaces, to which we refer as the first-order uncertainty space, second-order uncertainty space, and so on, and to an infinite hierarchy of preference orderings over his own strategies. In this hierarchy, the $k$-th order preference ordering at a given information set is induced by a subjective probability distribution (belief) over the $k$-th order uncertainty space and a utility function at reachable terminal nodes. In turn, the $k$-th order uncertainty space contains the opponents' possible $(k-1)$-th order preference relations, and hence a player, at each of his information sets, should hold a belief about the opponents' first-order, second-order, and higher order preference relations. In Perea (2003) it has been shown that the infinite preference hierarchies within our epistemic model can be handled efficiently by means of types. More precisely, it can be shown that each preference hierarchy of a player can be identified with a type, which specifies at each of the information sets a subjective probability distribution over the opponents' strategy choices and opponents' types, and which determines a utility function at the terminal nodes. This representation result thus justifies the use of a relatively simple, implicit type-model that makes the analysis easier.

We then proceed by imposing some restrictions upon the types, eventually leading to the concept of persistent rationalizability. The first two requirements, updating consistency and proper belief revision, are concerned with the belief updating and belief revision policies carried out by the types. Updating consistency simply states that Bayesian updating should be used whenever the observed behavior is still in accordance with the previously held beliefs. Proper belief revision states that, whenever a player $i$ type decides to revise his belief about player $j$ 's ranking of his own strategy choices, then he should not change his belief about $j$ 's relative

\footnotetext{
${ }^{1}$ Or, "highest possible degree of strategic sophistication", as Battigalli and Siniscalchi (2002) put it.
} 


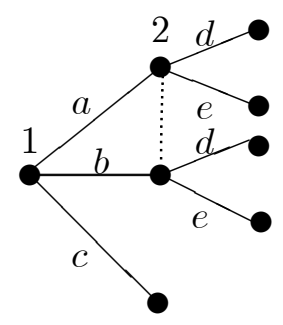

Figure 1

ranking of two strategies unless player $i$ is absolutely certain that $j$ has decided not to choose one of these strategies. The underlying principle is that a player should base his belief revision policy solely on the actual observed behavior, and not on conjectures concerning possible past and future behavior about which he is not absolutely certain. In order to illustrate this principle, consider Figure 1.

Suppose that player 2 initially believes that player 1 strictly prefers $c$ over $a$, and strictly prefers $a$ over $b$. If player 2 finds himself at his information set, he is certain that player 1 has not chosen $c$. If player 2 believes at this stage that player 1 has chosen rationally, player 2 is led to revise his belief about player 1's preference relation. Proper belief revision requires player 2 to maintain his belief that player 1 strictly prefers $a$ over $b$, while allowing player 1 to change his belief about the ranking of $c$ relative to $a$ and $b$. The reason is that the observed behavior, namely that player 1 has not chosen $c$, does not reveal new evidence about player $j$ 's ranking of $a$ relative to $b$, and therefore player 2 should not change his belief about this relative ranking.

The reason to call it proper belief revision is that this belief revision principle is implicitly present in the concepts of proper equilibrium (Myerson (1978)) and proper rationalizability (Schuhmacher (1999), Asheim (2001)). The key restriction in both concepts is that a player should never exclude any strategy choice by an opponent, yet should deem one opponent strategy "infinitely more likely" than another if he believes that the opponent strictly prefers the former above the latter. The notion of "infinitely more likely" can either be formalized by taking sequences of full-support probability distributions over the opponents' strategy choices and considering the relative likelihood of two strategy choices in the limit, as is done in Myerson (1978) and Schuhmacher (1999), or can be established by considering lexicographic probability distributions over strategy choices, as used in Blume, Brandenburger and Dekel (1991a, 1991b) and Asheim (2001) in their characterizations of proper equilibrium and proper rationalizability, respectively. If we would apply proper equilibrium or proper rationalizability to the situation in Figure 1, and assume that player 2 initially believes that player 1 strictly prefers $c$ over $a$, and strictly prefers $a$ over $b$, then player 2 should deem $c$ infinitely more likely than $a$, and deem $a$ infinitely more likely than $b$. Hence, if player 2 observes that player 1 has chosen $a$ or $b$, he should still deem $a$ infinitely more likely than $b$. Consequently, if player 2 is faced with the fact that player 1 has chosen $a$ or $b$, he should still believe that player 1 strictly prefers $a$ over $b$, and hence proper equilibrium and proper rationalizability imply the proper belief revision principle in this example. Since this argument can be applied to any given dynamic game, proper equilibrium 
and proper rationalizability generally support the proper belief revision principle.

The third condition we impose on types, belief in sequential rationality, reflects the principle that, whatever happens in the game, a player should always interpret observed moves as rational ones. In particular, if a player $i$ observes a move that would not have been optimal for an opponent $j$, were player $i$ to keep his previously held belief about $j$ 's utility function, then player $i$ should actually revise his belief about $j$ 's utilities in order to rationalize this move. Types that, throughout the game, respect common belief about the events that (1) types are updating consistent, (2) types satisfy proper belief revision, and (3) types believe in sequential rationality, are called persistently rationalizable.

The literature usually assumes some exogenously given restrictions upon the players' utility functions, and the beliefs they have about the opponents' utilities, modeled by the specification of a fixed profile of utility functions. The implicit interpretation is that players are assumed to hold these utility functions, and are to believe throughout the game that the opponents hold the utility functions as specified by the profile. As to link the concept of persistent rationalizability to existing rationality concepts for given utility functions, we subsequently impose some exogenous restrictions upon the players' utility functions and beliefs about the opponents' utilities. In order to do so, we proceed as above by taking as given a profile $u$ of utility functions, but a different interpretation shall now be attached to it. Players are required to hold the utility functions as specified by $u$, and to respect common belief about the event that players initially believe that opponents hold utility functions as given by $u$. Persistently rationalizable types that satisfy these additional requirements are said to be persistently rationalizable for $u$, and strategies that are optimal for such types at each of their information sets are called persistently rationalizable strategies for $u$. We thus leave open the possibility that players may change their belief about the opponents' utilities as the game is under way, while requiring that the players' beliefs agree on the same profile of utility functions at the beginning of the game.

In light of the latter property, our approach is related to the model of games with randomly disturbed payoffs, as used in Harsanyi (1973), Fudenberg, Kreps and Levine (1988), Dekel and Fudenberg (1990), Zauner (2002) and Stinchcombe and Zauner (2002), among others. In all of these papers, players are assumed to have "infinitesemal uncertainty" about the opponents' utility functions at the beginning of the game, modeled by a sequence of games with randomly perturbed utility functions in which the perturbation vanishes in the limit. The analysis then focusses on the behavior of players as the perturbation tends to zero. When applied to dynamic games, the choice of a sequence of utility perturbations may be seen as a way to model a particular belief revision policy for each player about the possible opponents' utilities. By letting the perturbation vanish in the limit, one imposes that the players' beliefs about the opponents' utilities should (approximately) agree on a particular profile of utility functions at the beginning of the game. A key factor that distinguishes our model from the ones above is that our proper belief revision condition imposes an explicit restriction upon the way players should revise their beliefs about the opponents' utilities, while the above mentioned papers, with the exception of Stinchcombe and Zauner (2002), put no constraints on the sequences of utility perturbations that may be chosen. ${ }^{2}$ Battigalli (2003), in his analysis of rationalizability in games

\footnotetext{
${ }^{2}$ Stinchcombe and Zauner (2002) do impose a restriction upon the sequence of utility perturbations, however it is different from proper belief revision.
} 
with "genuine incomplete information", takes another approach by assuming that players may have uncertainty about the other players' utility functions, without requiring that the players' beliefs at the beginning of the game agree on a particular profile of utility functions.

Having established the concept of persistent rationalizability for a given profile $u$ of utility functions, our next step is to present a refinement that focusses on types holding beliefs that are "as simple as possible". As to formalize the latter, we introduce the notion of the complexity of a type $t_{i}$, which, loosely speaking, represents the total number of types that $t_{i}$ considers directly or indirectly in his theory about the opponents. More precisely, the complexity of a type $t_{i}$ first counts the number of types $t_{j}$ that $t_{i}$ attaches positive probability to in his beliefs. For each of these types $t_{j}$, one counts the number of types that $t_{j}$ attaches positive probability to and that have not been counted already, and so on. By summing up all these types, one gets the total number of types that $t_{i}$ directly or indirectly refers to in his beliefs throughout the game, and this number is called the complexity of $t_{i}$. For a given profile of utility functions $u$, we say that a type is minimally rationalizable for $u$ if (1) it is persistently rationalizable for $u$, and (2) it has minimal complexity among all types that are persistently rationalizable for $u$. Accordingly, a strategy is called minimally rationalizable for $u$ if it can be chosen rationally by a type that is minimally rationalizable for $u$.

The second part of this paper is devoted to relating persistent and minimal rationalizability to existing rationality concepts in the literature. First of all, in Perea (2003) it has been shown that for every given profile $u$ of utility functions, every properly rationalizable strategy for $u$ for types with "non-increasing type supports" is persistently rationalizable for $u$. Here, the latter concept is a non-empty refinement of proper rationalizability, thus establishing the existence of persistently rationalizable types and strategies for all game trees and all utility functions $u$. Moreover, the proof of this result shows that persistently rationalizable types with finite complexity can always be found for all $u$, and hence minimally rationalizable types and strategies always exist.

We next find that persistent rationalizability may be viewed as a possible epistemic foundation for backward induction, since for generic games with perfect information the only persistently rationalizable strategy for each player is his backward induction strategy. Moreover, it establishes an interpretation of backward induction where moves off the backward induction path are not viewed as mistakes by one of the players, but rather as moves in accordance with common belief of rationality, while allowing players to adjust their beliefs about the opponents' utilities when observing such deviations from the backward induction path.

The concept of minimal rationalizability, on the other hand, turns out to have a strong forward induction flavour, at least in some classes of games. It is shown that in the class of outside option games, as introduced by van Damme (1989), the unique minimally rationalizable strategy for each player is his forward induction strategy. In particular, minimal rationalizability uniquely selects the forward induction outcome, which is the only Nash equilibrium outcome in the game that dominates the outside option from player 1's point of view. What is remarkable about this result is that no explicit equilibrium condition is needed in minimal rationalizability to filter this forward induction Nash equilibrium outcome.

The relation between minimal rationalizability and Nash equilibrium is even more transparent in the class of two-player simultaneous move games, since for such games the set of minimally 
rationalizable strategies for a player coincides exactly with the set of Nash equilibrium strategies. As such, minimal rationalizability provides an epistemic characterization of Nash equilibrium strategies for two-player static games. The major difference with the epistemic characterization provided by Aumann and Brandenburger (1995) is that minimal rationalizability does not explicitly impose mutual belief (or knowledge) concerning the players' beliefs about the opponent's strategy choice.

The outline of this paper is as follows. Section 2 presents some preliminary definitions in extensive form games. In Section 3 we present the epistemic framework that will be used as a basis for the rationalizability concepts. Section 4 lays out the concepts of persistent and minimal rationalizability. Sections 5,6 and 7 prove the above mentioned results on backward induction, Nash equilibrium in two-player simultaneous move games and outside option games, respectively. Section 8, finally, discusses the relationship with other rationalizability concepts such a weak sequential rationalizability and extensive form rationalizability.

\section{Extensive Form Structures}

In this section we introduce the necessary notation and some preliminary definitions for dynamic games. It is assumed that the reader is familiar with the precise definition of an extensive form game, which we therefore do not present in order to save space. Let $I$ be some finite set of players that faces a dynamic game. The rules of the game are given by an extensive form structure $\mathcal{S}$ consisting of (1) a finite game tree, (2) for every player $i$ some finite collection $H_{i}$ of information sets, (3) for every information set $h$ some finite set $A(h)$ of available actions, and (4) a finite set $Z$ of terminal nodes. We assume that the extensive form structure $\mathcal{S}$ satisfies perfect recall and contains no chance moves. The latter requirement is not really needed for our analysis, but rather simplifies the exposition. Let $H=\cup_{i \in I} H_{i}$ be the collection of all information sets in the game. By $h_{0}$ we denote the information set that marks the beginning of the game. Let $H_{i}^{*}=H_{i} \cup\left\{h_{0}\right\}$ for every player $i$.

The definition of a strategy we use in this paper follows Rubinstein's (1991) notion of a plan of action, specifying an action only at those information sets which are not avoided by the strategy itself. Let $\tilde{H}_{i} \subseteq H_{i}$ be some collection of player $i$ information sets, not necessarily including all, and let $s_{i}$ be a function that assigns an available action $s_{i}\left(h_{i}\right) \in A\left(h_{i}\right)$ to every information set $h_{i}$ in $\tilde{H}_{i}$. We say that an information set $h \in H$ (possibly controlled by some player other than $i$ ) is avoided by the function $s_{i}$ if every profile of available actions in $\times_{h \in H} A(h)$ that coincides with $s_{i}$ on $\tilde{H}_{i}$, avoids the information set $h$. The function $s_{i}$ defined on $\tilde{H}_{i}$ is called a strategy if $\tilde{H}_{i}$ is exactly the collection of player $i$ information sets not avoided by $s_{i}$. Obviously, every player $i$ strategy may be obtained by first prescribing an action at all player $i$ information sets, and then discarding those player $i$ information sets that are avoided by it. Let $S_{i}$ denote the set of player $i$ strategies.

An additional restriction we impose upon the extensive form structure is that it should be with observable deviators (see e.g. Battigalli (1996)). For its definition, we need some additional notation. For a given information set $h$, let $S(h)$ be the set of strategy profiles $\left(s_{i}\right)_{i \in I}$ that reach $h$, and let $S_{i}(h)$ be the set of player $i$ strategies that do not avoid $h$. Here, player $i$ needs not be the player who controls $h$. We say that the extensive form structure $\mathcal{S}$ is with observable 
deviators if $S(h)=\times_{i \in I} S_{i}(h)$ for every information set $h$. Hence, if every player $i$ chooses a strategy that cannot avoid $h$ by itself, then the resulting strategy profile will reach $h$. The condition is implied by perfect recall if there are only two players involved. For more than two players, however, this is no longer true.

\section{The Epistemic Model}

Our next step will be to lay out an epistemic model for dynamic games in which players, during the course of the game, may revise their beliefs about the opponents' preference relations, including the opponents' utilities at the terminal nodes. The model is identical to the one used in Perea (2003) and the reader is referred to that paper for the proofs of the results to be presented in this section. Before discussing the epistemic model, we shall briefly introduce the notions of acts and expected utility preference relations over acts, as they play a crucial role in the model.

Consider a compact metric space $X$ endowed with some topology. The space $X$ is to be interpreted as a collection of relevant parameters about which the decision maker is uncertain. Let $Y$ be some finite set of possible consequences, and $\Delta(Y)$ the set of probability distributions on $Y$, endowed with the natural topology. Following Anscombe and Aumann (1963), every decision may be identified with a mapping from $X$ to $\Delta(Y)$, to which we refer as acts. ${ }^{3}$ Let $\mathcal{F}(X, Y)$ be the set of measurable acts from $X$ to $\Delta(Y)$, and assume that the decision maker holds a preference relation over all acts in $\mathcal{F}(X, Y)$. We say that this preference relation is of the expected utility type if there is some probability distribution $\mu$ on $X$ and some von NeumannMorgenstern utility function $u$ from $Y$ to the real numbers such that act $f$ is weakly preferred to act $g$ if and only if

$$
\int_{X} u(f(x)) d \mu \geq \int_{X} u(g(x)) d \mu .
$$

Here, $u(f(x))$ is the expected utility induced by the probability distribution $f(x) \in \Delta(Y)$ and the utility function $u$. Similarly for $u(g(x))$. Let $\mathcal{P}^{e u}(X, Y)$ be the set of non-trivial expected utility preference relations on $\mathcal{F}(X, Y)$. For a given preference relation in $\mathcal{P}^{e u}(X, Y)$ the probability distribution $\mu$ is unique and the utility function is unique up to a positive affine transformation. Hence, every member of $\mathcal{P}^{e u}(X, Y)$ may be uniquely identified with a pair $(\mu, u)$ where $\mu$ is a probability distribution on $X$ and $u$ is a utility function on $Y$ with maximum value 1 and minimum value 0 . Let $U(Y)$ be the set of utility functions on $Y$ with the latter property. We thus may identify $\mathcal{P}^{e u}(X, Y)$ with the space $\Delta(X) \times U(Y)$. If we endow this space with the product toplogy induced by the weak topology on $\Delta(X)$ and the natural toplogy on $U(Y)$, the set $\mathcal{P}^{e u}(X, Y)$ becomes a compact metric space.

Let us now return to dynamic games. The basic assumption is that every player $i$, at each of his information sets $h_{i} \in H_{i}^{*}$, holds a preference relation on the set $S_{i}\left(h_{i}\right)$ of strategies that are compatible with $h_{i}$, while facing uncertainty about the opponents' strategy choices. Recall that $H_{i}^{*}=H_{i} \cup\left\{h_{0}\right\}$. At information set $h_{i}$, the set of feasible opponents' strategies is given by

\footnotetext{
${ }^{3}$ In Anscombe and Aumann (1963), such acts are called compound horse lotteries.
} 
$S_{-i}\left(h_{i}\right)=\times_{j \neq i} S_{j}\left(h_{i}\right)$. Hence, we may define

$$
X_{i}^{1}\left(h_{i}\right)=S_{-i}\left(h_{i}\right)
$$

as player $i$ 's first-order space of uncertainty at information set $h_{i}$. Let $Z\left(h_{i}\right)$ be the set of terminal nodes following $h_{i}$. Every strategy $s_{i}$ in $S_{i}\left(h_{i}\right)$ may now be identified with an act $f_{s_{i}}$ in $\mathcal{F}\left(S_{-i}\left(h_{i}\right), Z\left(h_{i}\right)\right)$ which assigns to every opponents' strategy profile $s_{-i} \in S_{-i}\left(h_{i}\right)$ the probability distribution on $Z\left(h_{i}\right)$ that attaches probability one to the terminal node reached by $\left(s_{i}, s_{-i}\right)$. Hence, $S_{i}\left(h_{i}\right)$ may be embedded in the set of acts $\mathcal{F}\left(X_{i}^{1}\left(h_{i}\right), Z\left(h_{i}\right)\right)$. It is assumed that player $i$ at $h_{i}$ holds a non-trivial expected utility preference relation $p_{i}^{1}\left(h_{i}\right)$ on all acts in $\mathcal{F}\left(X_{i}^{1}\left(h_{i}\right), Z\left(h_{i}\right)\right)$, that is, $p_{i}^{1}\left(h_{i}\right) \in \mathcal{P}^{e u}\left(X_{i}^{1}\left(h_{i}\right), Z\left(h_{i}\right)\right)$. We refer to $p_{i}^{1}\left(h_{i}\right)$ as player $i$ 's first-order preference relation at $h_{i}$, while $\mathcal{P}^{e u}\left(X_{i}^{1}\left(h_{i}\right), Z\left(h_{i}\right)\right)$ is the set of player $i$ 's first-order preference relations at $h_{i}$. By choosing the appropriate topology as described above, the latter set becomes a compact metric space.

The set $X_{i}^{1}\left(h_{i}\right)$, however, does not contain all relevant parameters about which player $i$ is uncertain at $h_{i}$, since player $i$ also faces uncertainty about the first-order preference relations that his opponents hold at each of their information sets. We may thus define player $i$ 's second-order space of uncertainty at $h_{i}$ by

$$
X_{i}^{2}\left(h_{i}\right)=X_{i}^{1}\left(h_{i}\right) \times\left(\times_{j \neq i} \times_{h_{j} \in H_{j}^{*}} \mathcal{P}^{e u}\left(X_{j}^{1}\left(h_{j}\right), Z\left(h_{j}\right)\right)\right),
$$

containing both the opponents' possible strategy choices that may have led to $h_{i}$ and the opponents' possible first-order preference relations at each of their information sets. Similarly as above, we assume that player $i$ at $h_{i}$ holds an expected utility preference relation $p_{i}^{2}\left(h_{i}\right)$ on the set of all acts in $\mathcal{F}\left(X_{i}^{2}\left(h_{i}\right), Z\left(h_{i}\right)\right)$. We refer to $p_{i}^{2}$ as player $i$ 's second-order preference relation at $h_{i}$, and to $\mathcal{P}^{e u}\left(X_{i}^{2}\left(h_{i}\right), Z\left(h_{i}\right)\right)$ as the set of possible second-order preference relations at $h_{i}$. The players' $k$-th order uncertainty spaces at their respective information sets may then be defined inductively by

$$
X_{i}^{k}\left(h_{i}\right)=X_{i}^{k-1}\left(h_{i}\right) \times\left(\times_{j \neq i} \times_{h_{j} \in H_{j}^{*}} \mathcal{P}^{e u}\left(X_{j}^{k-1}\left(h_{j}\right), Z\left(h_{j}\right)\right)\right)
$$

for all players $i$, information sets $h_{i} \in H_{i}^{*}$ and $k \geq 2$. At each information set $h_{i}$, player $i$ is assumed to hold a hierarchy of preference relations $p_{i}\left(h_{i}\right)=\left(p_{i}^{k}\left(h_{i}\right)\right)_{k \in \mathbb{N}}$, where $p_{i}^{k}\left(h_{i}\right)$ represents the $k$-th order preference relation in $\mathcal{P}^{e u}\left(X_{i}^{k}\left(h_{i}\right), Z\left(h_{i}\right)\right)$. A vector $p_{i}=\left(p_{i}\left(h_{i}\right)\right)_{h_{i} \in H_{i}^{*}}$ of such hierarchies of preference relations, one for each information set, is simply called a preference hierarchy for player $i$. Let $P_{i}$ be the set of all preference hierarchies for player $i$.

Similar to Epstein and Wang (1996), a preference hierarchy $p_{i}$ is called coherent if for every information set $h_{i}$ and every order $k \geq 2$, the marginal of the $k$-th order preference relation $p_{i}^{k}\left(h_{i}\right)$ on the $(k-1)$-th order space of acts $\mathcal{F}\left(X_{i}^{k-1}\left(h_{i}\right), Z\left(h_{i}\right)\right)$ coincides with the $(k-1)$-th order preference relation $p_{i}^{k-1}\left(h_{i}\right) .{ }^{4}$ In words, coherence means that the different preference relations in the hierarchy should coincide on overlapping subspaces. We obtain the following representation result for coherent preference hierarchies, for which a proof can be found in Perea (2003).

\footnotetext{
${ }^{4}$ Every act in $\mathcal{F}\left(X_{i}^{k-1}\left(h_{i}\right), Z\left(h_{i}\right)\right)$ may be identified with some act in $\mathcal{F}\left(X_{i}^{k}\left(h_{i}\right), Z\left(h_{i}\right)\right)$ that only depends on the argument in $X_{i}^{k-1}\left(h_{i}\right)$. As such, $\mathcal{F}\left(X_{i}^{k-1}\left(h_{i}\right), Z\left(h_{i}\right)\right)$ may be viewed as a subspace of $\mathcal{F}\left(X_{i}^{k}\left(h_{i}\right), Z\left(h_{i}\right)\right)$, and hence the marginal of $p_{i}^{k}\left(h_{i}\right)$ on $\mathcal{F}\left(X_{i}^{k-1}\left(h_{i}\right), Z\left(h_{i}\right)\right)$ is well-defined.
} 
Lemma 3.1. For every player $i$, the set of coherent preference hierarchies is homeomorphic to the space $\times_{h_{i} \in H_{i}^{*}} \mathcal{P}^{e u}\left(S_{-i}\left(h_{i}\right) \times P_{-i}, Z\left(h_{i}\right)\right)$.

Here, $P_{-i}=\times_{j \neq i} P_{j}$ is the space of all opponents' preference hierarchies. As such, every coherent preference hierarchy $p_{i}$ for player $i$ induces at every information set $h_{i} \in H_{i}^{*}$ some expected utility preference relation on acts in $\mathcal{F}\left(S_{-i}\left(h_{i}\right) \times P_{-i}, Z\left(h_{i}\right)\right)$, representable by a probability distribution $\mu_{i}\left(p_{i}, h_{i}\right)$ on $S_{-i}\left(h_{i}\right) \times P_{-i}$, and some utility function $u_{i}\left(p_{i}, h_{i}\right)$ on $Z\left(h_{i}\right)$. For an opponent $j$, let $\mu_{i}\left(p_{i}, h_{i} \mid P_{j}\right)$ be the marginal of the probability distribution $\mu_{i}\left(p_{i}, h_{i}\right)$ on the set of player $j$ 's preference hierarchies. Let $P_{j}\left(p_{i}, h_{i}\right)=\operatorname{supp} \mu_{i}\left(p_{i}, h_{i} \mid P_{j}\right)$ be the set of player $j$ 's preference hierarchies to which $p_{i}$ assigns positive probability at $h_{i}$. For $j=i$, we define $P_{i}\left(p_{i}, h_{i}\right)=\left\{p_{i}\right\}$. By $P\left(p_{i}, h_{i}\right)=\cup_{j \in I} P_{j}\left(p_{i}, h_{i}\right)$ we denote the set of all preference hierarchies to which $p_{i}$ assigns positive probability at $h_{i}$. Let $P\left(p_{i}\right)=\cup_{h_{i} \in H_{i}^{*}} P\left(p_{i}, h_{i}\right)$ be the set of all preference hierarchies to which $p_{i}$ assigns positive probability somewhere in the game.

Let $\tilde{P} \subseteq \cup_{j \in I} P_{j}$ be a set of profiles of preference hierarchies, or simply an event. We say that the coherent preference hierarchy $p_{i}$ believes the event $\tilde{P}$ if $P\left(p_{i}\right) \subseteq \tilde{P}$, that is, if $p_{i}$ only assigns positive probability to preference hierarchies that belong to $\tilde{P}$. We now define common belief about coherence by means of the following recursive definition of sets: let $P_{i}^{c, 1}$ be the set of coherent preference hierarchies for player $i$, and for every $k \geq 2$ let $P_{i}^{c, k}$ be the set of preference hierarchies in $P_{i}^{c, k-1}$ that believe $\cup_{j \in I} P_{j}^{c, k-1}$. By $P_{i}^{c, \infty}=\cap_{k \in \mathbb{N}} P_{i}^{c, k}$ we denote the set of preference hierarchies that respect common belief about coherence. Hence, $p_{i} \in P_{i}^{c, \infty}$ if and only if $p_{i}$ is coherent, believes that all opponents' preference hierarchies are coherent, believes that all opponents' preference hierarchies believe that all other players' preference hierarchies are coherent, and so on. In the spirit of Armbruster and Böge (1979), Böge and Eisele (1979) and Mertens and Zamir (1985) we may now derive the following representation result for preference hierarchies that respect common belief about coherence. A proof may be found in Perea (2003).

Lemma 3.2. For every player $i$, the set $P_{i}^{c, \infty}$ of preference hierarchies that respect common belief of coherence is homeomorphic to the space $\times_{h_{i} \in H_{i}^{*}} \mathcal{P}^{e u}\left(S_{-i}\left(h_{i}\right) \times P_{-i}^{c, \infty}, Z\left(h_{i}\right)\right)$.

By $T_{i}=P_{i}^{c, \infty}$ we denote the set of player $i$ types. Then, by the lemma above, the type-space $T_{i}$ for every player $i$ is homeomorphic to $\times_{h_{i} \in H_{i}^{*}} \mathcal{P}^{e u}\left(S_{-i}\left(h_{i}\right) \times T_{-i}, Z\left(h_{i}\right)\right)$. That is, every type $t_{i}$ may be identified with a vector that induces at every information set $h_{i} \in H_{i}^{*}$ a probability distribution, or belief, $\mu_{i}\left(t_{i}, h_{i}\right)$ on $S_{-i}\left(h_{i}\right) \times T_{-i}$, and a utility function $u_{i}\left(t_{i}, h_{i}\right)$ on $Z\left(h_{i}\right)$. Similarly as we have done above for coherent preference hierarchies $p_{i}$, we may define for every type $t_{i}$ the set $T\left(t_{i}\right) \subseteq \cup_{j \in I} T_{j}$ as the set of types to which $t_{i}$ assigns positive probability somewhere in the game. We recursively define the sets of types $T^{1}\left(t_{i}\right), T^{2}\left(t_{i}\right), \ldots$ by $T^{1}\left(t_{i}\right)=$ $T\left(t_{i}\right)$, and

$$
T^{k}\left(t_{i}\right)=\bigcup_{t \in T^{k-1}\left(t_{i}\right)} T(t)
$$

for all $k \geq 2$. By construction, $T^{k}\left(t_{i}\right)$ for $k \geq 2$ is the set of types $t$ for which one can build a sequence $\left(t_{i}=t^{1}, t^{2}, \ldots, t^{k}=t\right)$ of length $k$ such that $t_{i}$ assigns positive probability to $t^{2}$, $t^{2}$ assigns positive probability to $t^{3}, \ldots, t^{k-1}$ assigns positive probability to $t$ somewhere in the 
game. By $T^{\infty}\left(t_{i}\right)=\cup_{k \in \mathbb{N}} T^{k}\left(t_{i}\right)$ we denote the set of types that may be reached from $t_{i}$ by building such sequences of arbitrary length.

Let $\tilde{T} \subseteq \cup_{j \in I} T_{j}$ be a collection of types, or simply an event. We say that type $t_{i}$ respects common belief about $\tilde{T}$ if $T^{\infty}\left(t_{i}\right) \subseteq \tilde{T}$. In words, $t_{i}$ believes that all types belong to $\tilde{T}$, believes that all types believe that all types belong to $\tilde{T}$, and so on.

\section{Persistent and Minimal Rationalizability}

\subsection{Persistent Rationalizability}

We shall now impose three restrictions upon types in our epistemic model: updating consistency, proper belief revision and belief in sequential rationality. The concept of persistent rationalizability then selects those types that respect common belief about these three events throughout the game.

Updating consistency requires a type to update his beliefs using Bayes' rule, whenever possible.

Definition 4.1. A type $t_{i}$ is called updating consistent if for every two information sets $h_{i}^{1}, h_{i}^{1} \in$ $H_{i}^{*}$ such that $h_{i}^{2}$ follows $h_{i}^{1}$, and every event $E \subseteq S_{-i}\left(h_{i}^{2}\right) \times T_{-i}$, it holds that

$$
\mu_{i}\left(t_{i}, h_{i}^{2}\right)(E)=\frac{\mu_{i}\left(t_{i}, h_{i}^{1}\right)(E)}{\mu_{i}\left(t_{i}, h_{i}^{1}\right)\left(S_{-i}\left(h_{i}^{2}\right) \times T_{-i}\right)}
$$

whenever $\mu_{i}\left(t_{i}, h_{i}^{1}\right)\left(S_{-i}\left(h_{i}^{2}\right) \times T_{-i}\right)>0$.

Proper belief revision states that a type, when revising his belief about an opponent's preference relation, should not change his belief about the opponent's relative ranking of two strategies unless he is certain that the opponent has not chosen one of these strategies. Formally, let $t_{i}$ be a type and $h_{i} \in H_{i}^{*}$ an information set for player $i$. By definition, $t_{i}$ knows at $h_{i}$ that opponent $j$ has chosen some strategy in $S_{j}\left(h_{i}\right)$, without being able to exclude any of these strategies. Proper belief revision then argues that $t_{i}$ may revise his belief at $h_{i}$ about player $j$ 's preference relation over strategies in $S_{j}$, but should not change his belief about player $j$ 's ranking of strategies in $S_{j}\left(h_{i}\right)$. In the formal definition below, let $T_{j}\left(t_{i}, h_{i}\right)$ be the set of player $j$ types to which $t_{i}$ assigns positive probability at information set $h_{i}$.

Definition 4.2. A type $t_{i}$ is said to satisfy proper belief revision if for every two information sets $h_{i}^{1}, h_{i}^{2} \in H_{i}^{*}$ with $h_{i}^{2}$ following $h_{i}^{1}$, and every type $t_{j}^{2} \in T_{j}\left(t_{i}, h_{i}^{2}\right)$, there is some type $t_{j}^{1} \in$ $T_{j}\left(t_{i}, h_{i}^{1}\right)$ with the property that $t_{j}^{1}$ and $t_{j}^{2}$ hold the same preference relation over strategies in $S_{j}\left(h_{i}^{2}\right) \cap S_{j}\left(h_{j}\right)$ at every information set $h_{j} \in H_{j}^{*}$.

Finally, belief in sequential rationality reflects the principle that a player, at each of his information sets, should believe that his opponents are carrying out strategies that are optimal for them at each of their information sets. Formally, we say that a strategy-type pairs $\left(s_{i}, t_{i}\right)$ is sequentially rational if at every information set $h_{i} \in H_{i}^{*}\left(s_{i}\right)$ it holds that

$$
u_{i}\left(t_{i}, t_{i} \mid h_{i}\right)=\max _{s_{i}^{\prime} \in S_{i}\left(h_{i}\right)} u_{i}\left(s_{i}^{\prime}, t_{i} \mid h_{i}\right) .
$$


Here, $H_{i}^{*}\left(s_{i}\right)$ denotes the collection of information sets in $H_{i}^{*}$ that are not avoided by $s_{i}$. By $u_{i}\left(t_{i}, t_{i} \mid h_{i}\right)$ we denote the expected utility at $h_{i}$ induced by the utility function $u_{i}\left(t_{i}, h_{i}\right)$, the strategy $s_{i}$ and the marginal of the belief $\mu_{i}\left(t_{i}, h_{i}\right)$ on the set $S_{-i}\left(h_{i}\right)$ of opponents' strategies. For every player $j$, let $\left(S_{j} \times T_{j}\right)^{s r}$ be the set of sequentially rational strategy-type pairs. For a given player $i$, let $\left(S_{-i} \times T_{-i}\right)^{s r}=\times_{j \neq i}\left(S_{j} \times T_{j}\right)^{s r}$ be the set of opponents' sequentially rational strategy-type pairs.

Definition 4.3. A type $t_{i}$ is said to believe in sequential rationality if for every information set $h_{i} \in H_{i}^{*}$ it holds that $\operatorname{supp} \mu_{i}\left(t_{i}, h_{i}\right) \subseteq\left(S_{-i} \times T_{-i}\right)^{s r}$.

We are now ready to define the concept of persistent rationalizability.

Definition 4.4. A type $t_{i}$ is called persistently rationalizable if it respects common belief about the events that (1) types are updating consistent, (2) types satisfy proper belief revision, and (3) types believe in sequential rationality.

\subsection{Restrictions on Utility Functions and Initial Beliefs}

We now proceed by imposing some exogenous restrictions upon the players' actual utility functions, and the initial beliefs players have about the utility functions of others. Consider a profile $u=\left(u_{i}\right)_{i \in I}$ of utility functions over the terminal nodes. Together with the extensive form structure $\mathcal{S}$, this induces a pair $(\mathcal{S}, u)$ which is usually called an extensive form game. The most common interpretation of $(\mathcal{S}, u)$ is that players hold utility functions as specified by $u$, and that they should believe throughout the game that the opponents have utility functions as given by $u$. Our interpretation of $(\mathcal{S}, u)$ will be different since we require players to initially believe that opponents have utility functions in $u$, while allowing the players to change their beliefs about the opponents' utility functions later on in the game. Formally, we say that a type $t_{i}$ initially believes $u$ if supp $\mu_{i}\left(t_{i}, h_{0}\right)$ only contains types $t_{j}$ with $u_{j}\left(t_{j}, h_{j}\right)=\left.u_{j}\right|_{Z\left(h_{j}\right)}$ for all $h_{j} \in H_{j}^{*}$. Here, $\left.u_{j}\right|_{Z\left(h_{j}\right)}$ denotes the restriction of $u_{j}$ on the set $Z\left(h_{j}\right)$ of terminal nodes following $h_{j}$.

Definition 4.5. Let the profile $u=\left(u_{i}\right)_{i \in I}$ of utility functions be given. We say that a type $t_{i}$ is persistently rationalizable for $(\mathcal{S}, u)$ if $(1) t_{i}$ is persistently rationalizable, $(2) u_{i}\left(t_{i}, h_{i}\right)=\left.u_{i}\right|_{Z\left(h_{i}\right)}$ for all $h_{i} \in H_{i}^{*}$, and (3) $t_{i}$ respects common belief about the event that types initially believe $u$. We say that a strategy $s_{i}$ is persistently rationalizable for $(\mathcal{S}, u)$ if there is a persistently rationalizable type $t_{i}$ for $(\mathcal{S}, u)$ such that $\left(s_{i}, t_{i}\right)$ is sequentially rational.

\subsection{Minimal Rationalizability}

We shall next focus on types that are persistently rationalizable for a given extensive form game $(\mathcal{S}, u)$, and, moreover, hold a theory about the opponents that in some sense is "as simple as possible". In order to formalize the latter, we introduce the notion of the complexity of a type. Recall from Section 3 that for a given type $t_{i}$, the set $T^{\infty}\left(t_{i}\right) \subseteq \cup_{j \in I} T_{j}$ denotes the collection of types $t_{j}$ such that either (1) $t_{i}$ assigns positive probability to $t_{j}$ at one of his information sets, (2) $t_{i}$ assigns positive probability to some type $t_{k}$ which assigns positive probability to $t_{j}$ at some of his information sets, and so on. Hence, one could say that $T^{\infty}\left(t_{i}\right)$ represents the set 
of types that $t_{i}$ uses, directly or indirectly, in his theory about the opponents' strategies and beliefs throughout the game. Let $c\left(t_{i}\right)$ be the total number of types in $T^{\infty}\left(t_{i}\right)$, which could in principle be infinite. We refer to $c\left(t_{i}\right)$ as the complexity of type $t_{i}$.

Definition 4.6. Let $(\mathcal{S}, u)$ be an extensive form game. Then, a type $t_{i}$ is called minimally rationalizable for $(\mathcal{S}, u)$ if $t_{i}$ is persistently rationalizable for $(\mathcal{S}, u)$ and has minimal complexity among all player $i$ types that are persistently rationalizable for $(\mathcal{S}, u)$. A strategy $s_{i}$ is said to be minimally rationalizable for $(\mathcal{S}, u)$ if there is some minimally rationalizable type $t_{i}$ for such that $\left(s_{i}, t_{i}\right)$ is sequentially rational.

\subsection{Existence}

In Perea (2003) it has been shown that for every extensive form structure $\mathcal{S}$ with observable deviators, and every profile $u$ of utility functions, every player has at least one persistently rationalizable type and strategy for $(\mathcal{S}, u)$. The existence follows from a theorem establishing a general relationship between the concept of proper rationalizability (Schuhmacher (1999) and Asheim (2001)) on the one hand, and the concept of persistent rationalizability on the other hand. More precisely, the theorem states that for a given extensive form game $(\mathcal{S}, u)$, every properly rationalizable strategy for $(\mathcal{S}, u)$ for types with "non-inceasing type-supports" is a persistently rationalizable strategy for $(\mathcal{S}, u)$. The former notion constitutes a refinement of proper rationalizability, and following Asheim (2001) it can easily be shown that properly rationalizable strategies for types with non-increasing type supports always exist for every $(\mathcal{S}, u)$.

The proof of the theorem not only establishes the existence of persistently rationalizable types for all $(\mathcal{S}, u)$, it also shows that we can always find a persistently rationalizability type with finite complexity for each player. The reason is that the proof chooses for every $(\mathcal{S}, u)$ a properly rationalizable type with "non-increasing type supports" (this can always be found) and then explicitly transforms this type into a persistently rationalizable type for $(\mathcal{S}, u)$ with finite complexity. As such, the notion of minimal rationalizability for $(\mathcal{S}, u)$ is always well-defined, and every minimally rationalizable type will always have a finite complexity. In the remainder of this paper, we shall apply the concepts of persistent and minimal rationalizability to several special classes of games, and investigate their relationships to existing rationality concepts.

\section{Games with Perfect Information}

In this section we show that in generic games with perfect information, every player has a unique persistently rationalizable strategy, namely his backward induction strategy. A game with perfect information $(\mathcal{S}, u)$ is said to be in generic position if for every player $i$ and every pair $z_{1}, z_{2}$ of different terminal nodes, we have that $u_{i}\left(z_{1}\right) \neq u_{i}\left(z_{2}\right)$. For such a game, let $a^{*}\left(h_{i}\right) \in A\left(h_{i}\right)$ denote the unique backward induction action at information set $h_{i}$. For every player $i$, there is a unique strategy $s_{i}^{*}$ with $s_{i}^{*}\left(h_{i}\right)=a^{*}\left(h_{i}\right)$ for all $h_{i} \in H_{i}\left(s_{i}^{*}\right)$, to which we shall refer as the backward induction strategy.

Theorem 5.1. Let $(\mathcal{S}, u)$ be a game with perfect information in generic position. Then, a strategy is persistently rationalizable for $(\mathcal{S}, u)$ if and only if it is a backward induction strategy for $(\mathcal{S}, u)$. 
Proof. Let $(\mathcal{S}, u)$ be a game with perfect information in generic position. For every player $i$ and every information set $h_{i}$, let $S_{i}^{*}\left(h_{i}\right)$ denote the set of strategies $s_{i}$ such that (1) at every information set $\tilde{h}_{i} \in H_{i}$ preceding $h_{i}$ the strategy $s_{i}$ prescribes the unique action at $\tilde{h}_{i}$ which leads to $h_{i}$, and $(2)$ at every information set $\tilde{h}_{i}$ following $h_{i}$ which is not avoided by $s_{i}$, the strategy $s_{i}$ prescribes the unique backward induction action $a^{*}\left(h_{i}\right)$. We refer to $S_{i}^{*}\left(h_{i}\right)$ as the set of player $i$ backward induction strategies conditional on $h_{i}$ in the game $(\mathcal{S}, u)$. Let $T_{i}^{*}\left(h_{i}\right)$ be the set of player $i$ types $t_{i}$ such that $t_{i}$ 's most preferred strategies at $h_{i}$ all belong to $S_{i}^{*}\left(h_{i}\right)$. For a given type $t_{i}$, let $\mu_{i}\left(t_{i}, h_{i} \mid T_{j}\right)$ be the marginal probability distribution of $\mu_{i}\left(t_{i}, h_{i}\right)$ on player $j$ 's types. Let $T_{i}^{\infty}(u)$ be the set of player $i$ types that respect common belief about the event that types initially believe $u$. We prove the following claim.

Claim. Let $t_{i}$ be persistently rationalizable and $t_{i} \in T_{i}^{\infty}(u)$. Then, for every information set $h_{i} \in H_{i}^{*}$, every opponent $j$ and information set $h_{j}$ following $h_{i}$, it holds that $\operatorname{supp} \mu_{i}\left(t_{i}, h_{i} \mid\right.$ $\left.T_{j}\right) \subseteq T_{j}^{*}\left(h_{j}\right)$.

Proof of claim. By induction on the number of decision nodes following $h_{j}$. Suppose first that $h_{j}$ is not followed by any decision node. Let $t_{j} \in \operatorname{supp} \mu_{i}\left(t_{i}, h_{i} \mid T_{j}\right)$. Then, since $t_{i}$ satisfies proper belief revision, there is some $t_{j}^{0} \in \operatorname{supp} \mu_{i}\left(t_{i}, h_{0} \mid T_{j}\right)$ such that $t_{j}^{0}$ and $t_{j}$ have at $h_{j}$ the same preference relation over strategies in $S_{j}\left(h_{i}\right)$. Since $h_{i}$ precedes $h_{j}$, we have that $S_{j}\left(h_{j}\right) \subseteq S_{j}\left(h_{i}\right)$, and hence $t_{j}^{0}$ and $t_{j}$ have the same preference relation over strategies in $S_{j}\left(h_{j}\right)$. Since $t_{i} \in T_{i}^{\infty}(u)$ we have that $t_{i}$ initially believes $u$, and hence $u_{j}\left(t_{j}^{0}, h_{j}\right)=\left.u_{j}\right|_{Z\left(h_{j}\right)}$. Since $h_{j}$ is not followed by any decision node, it must hold that $t_{j}^{0}$ at $h_{j}$ strictly prefers the backward induction action. But then, since $t_{j}$ at $h_{j}$ has the same preference relation over actions at $h_{j}$ as $t_{j}^{0}$, it must hold that also $t_{j}$ at $h_{j}$ strictly prefers the backward induction action, and hence $t_{j} \in T_{j}^{*}\left(h_{j}\right)$. We thus have shown that $\operatorname{supp} \mu_{i}\left(t_{i}, h_{i} \mid T_{j}\right) \subseteq T_{j}^{*}\left(h_{j}\right)$.

Now, suppose that the claim holds for every $t_{i}, h_{i}$ and $h_{j}$ where $h_{j}$ is followed by at most $K-1$ decision nodes. We prove the claim for information sets $h_{j}$ followed by $K$ decision nodes. Choose $h_{i}$ and $h_{j}$ such that $h_{j}$ follows $h_{i}$ and $h_{j}$ is followed by $K$ decision nodes. Let $t_{j} \in \operatorname{supp} \mu_{i}\left(t_{i}, h_{i} \mid T_{j}\right)$. We show that $t_{j} \in T_{j}^{*}\left(h_{j}\right)$. Since $t_{i}$ is persistently rationalizable and $t_{i} \in T_{i}^{\infty}(u)$, we have that $t_{j}$ is persistently rationalizable and $t_{j} \in T_{j}^{\infty}(u)$. Let player $k \neq j$ and information set $h_{k} \in H_{k}$ be such that $h_{k}$ follows $h_{j}$. Since $h_{k}$ is followed by at most $K-1$ decision nodes, we may apply the induction assumption to $t_{j}, h_{j}$ and $h_{k}$ and conclude that $\operatorname{supp} \mu_{j}\left(t_{j}, h_{j} \mid\right.$ $\left.T_{k}\right) \subseteq T_{k}^{*}\left(h_{k}\right)$. Hence, for all players $k \neq j$ and all information sets $h_{k}$ following $h_{j}$ we have that $\operatorname{supp} \mu_{j}\left(t_{j}, h_{j} \mid T_{k}\right) \subseteq T_{k}^{*}\left(h_{k}\right)$. Since $t_{j}$ is persistently rationalizable, we have in particular that $t_{j}$ believes in sequential rationality. By the above, we may thus conclude that $t_{j}$ believes at $h_{j}$ that at all future information sets the corresponding player chooses the backward induction action.

Since $t_{j} \in \operatorname{supp} \mu_{i}\left(t_{i}, h_{i} \mid T_{j}\right)$ and $t_{i}$ satisfies proper belief revision, there is some $t_{j}^{0} \in$ $\operatorname{supp} \mu_{i}\left(t_{i}, h_{0} \mid T_{j}\right)$ such that $t_{j}^{0}$ and $t_{j}$ hold the same preference relation over strategies in $S_{j}\left(h_{i}\right) \supseteq S_{j}\left(h_{j}\right)$. As $t_{i}$ initially believes $u$, it must hold that $u_{j}\left(t_{j}^{0}, h_{j}\right)=\left.u_{j}\right|_{Z\left(h_{j}\right)}$. Moreover, since $t_{j}^{0} \in \operatorname{supp} \mu_{i}\left(t_{i}, h_{0} \mid T_{j}\right)$ we may apply the same reasoning as above to conclude that $t_{j}^{0}$ is persistently rationalizable and $t_{j}^{0} \in T_{j}^{\infty}(u)$. But then, by copying the argument above for $t_{j}$, this leads to the conclusion that $t_{j}^{0}$ believes at $h_{j}$ that at all future information sets the corresponding player chooses the backward induction action. Together with the fact that $u_{j}\left(t_{j}^{0}, h_{j}\right)=\left.u_{j}\right|_{Z\left(h_{j}\right)}$, this implies that $t_{j}^{0}$ 's most preferred strategies at $h_{j}$ are backward induction strategies conditional 
on $h_{j}$.

Above we have seen that $t_{j}^{0}$ and $t_{j}$ hold the same preference relation over strategies in $S_{j}\left(h_{j}\right)$, and hence $t_{j}$ 's most preferred strategies at $h_{j}$ are backward induction strategies conditional on $h_{j}$, that is, $t_{j} \in T_{j}^{*}\left(h_{j}\right)$. This completes the proof of the claim.

Now, choose an arbitrary strategy $s_{i}$ that is persistently rationalizable for $(\mathcal{S}, u)$. Then, there must be a type $t_{i}$ which is persistently rationalizable for $(\mathcal{S}, u)$ such that $s_{i}$ is sequentially rational for $t_{i}$. By definition, $t_{i} \in T_{i}^{\infty}(u)$, and hence, by the claim, it follows that for every information set $h_{i} \in H_{i}^{*}$, every opponent $j$ and information set $h_{j}$ following $h_{i}$, we have $\operatorname{supp} \mu_{i}\left(t_{i}, h_{i} \mid\right.$ $\left.T_{j}\right) \subseteq T_{j}^{*}\left(h_{j}\right)$. Since $t_{i}$ believes in sequential rationality, we may conclude that $t_{i}$ believes at every information set $h_{i}$ that at all future information sets the corresponding player chooses his backward induction action. By assumption, $u_{i}\left(t_{i}, h_{i}\right)=\left.u_{i}\right|_{Z\left(h_{i}\right)}$ for all information sets $h_{i}$, and hence $t_{i}$ prefers at every information set $h_{i}$ a backward induction strategy conditional on $h_{i}$. Since $s_{i}$ is sequentially rational for $t_{i}$, it must be that $s_{i}$ is player $i$ 's unique backward induction strategy in $(\mathcal{S}, u)$. This completes the proof of this theorem.

In view of Theorem 5.1, the concept of persistent rationalizability may be employed as an alternative epistemic foundation for backward induction in games with perfect information. There is an important difference with other foundations proposed in the literature, such as Aumann (1995), Samet (1996), Balkenborg and Winter (1997), Stalnaker (1998) and Asheim (2000), as persistent rationalizability allows players to revise their conjectures about the opponents' utility functions during the game, whereas the latter foundations do not. In turn, persistent rationalizability requires players to interpret "unexpected moves" (in this case, moves that deviate from the backward induction play) always as being in accordance with common belief of rationality.

\section{Simultaneous Move Games}

In Section 4, we have defined minimally rationalizable types for $(\mathcal{S}, u)$ as those persistently rationalizable types for $(\mathcal{S}, u)$ that have minimal complexity. Recall that the complexity of a type $t_{i}$ denotes the total number of types that $t_{i}$, directly or indirectly, uses in his theory about the opponents' beliefs. In this section, we show that the minimal complexity criterion has non-trivial implications even for the class of simultaneous move games in which belief revision plays no role. In these games, persistent rationalizability is equivalent to rationalizability, as defined in Bernheim (1984) and Pearce (1984). Together with the restriction that types hold utility functions as specified by $u$ and that there be common belief about the event that types initially believe $u$, the epistemic model of Section 3, when applied to simultaneous move games, is equivalent to the one used by Tan and Werlang (1988). Minimal rationalizability thus restricts attention to those rationalizable strategies that can be justified by an epistemic rationalizability theory (cf. Tan and Werlang (1988)) which involves as few types as possible. We shall prove that for the case of two-player simultaneous move games, this concept is equivalent to the notion of Nash equilibrium strategies.

In order to formalize this result, we first need the definition of a Nash equilibrium strategy. For a given two-person simultaneous move game, a first-order belief about player $i$ is a probability distribution $\mu_{i} \in \Delta\left(S_{i}\right)$, reflecting player $j$ 's belief about player $i$ 's strategy choice. A profile 
$\left(\mu_{1}, \mu_{2}\right)$ of first-order beliefs is a Nash equilibrium if $\mu_{i}\left(s_{i}\right)>0$ implies that $s_{i}$ is a best response against $\mu_{j}$. A strategy $s_{i}$ is a Nash equilibrium strategy if there is some Nash equilibrium $\left(\mu_{1}, \mu_{2}\right)$ such that $s_{i}$ is a best response against $\mu_{j}$. Since not every rationalizable strategy in a two-player game is a Nash equilibrium strategy, the following result implies that minimal rationalizability is indeed stronger than rationalizability in two-player simultaneous move games.

Theorem 6.1. Let $(\mathcal{S}, u)$ be a two-player simultaneous move game. Then, $s_{i}$ is minimally rationalizable for $(\mathcal{S}, u)$ if and only if $s_{i}$ is a Nash equilibrium strategy for $(\mathcal{S}, u)$.

Proof. Let $s_{i}$ be a Nash equilibrium strategy. Then, there is some Nash equilibrium $\left(\mu_{i}, \mu_{j}\right)$ in first-order beliefs such that $s_{i}$ is optimal against $\mu_{j}$. We may construct two types $t_{i}$ and $t_{j}$ such that $u_{i}\left(t_{i}, h_{0}\right)=u_{i}, \mu_{i}\left(t_{i}, h_{0}\right)$ assigns probability one to $t_{j}, \mu_{i}\left(t_{i}, h_{0}\right)\left(s_{j}, t_{j}\right)=\mu_{j}\left(s_{j}\right)$ for all $s_{j} \in S_{j}$, and similarly for type $t_{j}$. Then, by the properties of Nash equilibrium, $t_{i}$ is persistently rationalizable. Since $T^{\infty}\left(t_{i}\right)=\left\{t_{i}, t_{j}\right\}$, the complexity of $t_{i}$ is 2 , which is clearly minimal. Hence, $t_{i}$ is minimally rationalizable. Since $s_{i}$ is optimal against $\mu_{j}$, it follows that $\left(s_{i}, t_{i}\right)$ is sequentially rational, which implies that $s_{i}$ is minimally rationalizable for $(\mathcal{S}, u)$.

Now, let $s_{i}$ be minimally rationalizable. Then, there exists some minimally rationalizable type $t_{i}$ for this game such that $\left(s_{i}, t_{i}\right)$ is sequentially rational. We have seen above that every Nash equilibrium induces a type that is persistently rationalizable for $(\mathcal{S}, u)$ with complexity 2 . Since Nash equilibria always exist, we may thus conclude that $t_{i}$ must have complexity 2 , that is, $T^{\infty}\left(t_{i}\right)=\left\{t_{i}, t_{j}\right\}$ for some $t_{j}$. Hence, $\mu_{i}\left(t_{i}, h_{0}\right)$ assigns probability one to $t_{j}$, and $\mu_{j}\left(t_{j}, h_{0}\right)$ assigns probability one to $t_{i}$. Let $\mu_{i}$ be the marginal of $\mu_{j}\left(t_{j}, h_{0}\right)$ on $S_{i}$, and $\mu_{j}$ the marginal of $\mu_{i}\left(t_{i}, h_{0}\right)$ on $S_{j}$. Since $t_{i}$ is persistently rationalizable, it follows that $\left(\mu_{i}, \mu_{j}\right)$ is a Nash equilibrium. Since $\left(s_{i}, t_{i}\right)$ is sequentially rational, it follows that $s_{i}$ is optimal against $\mu_{j}$, and hence $s_{i}$ is a Nash equilibrium strategy. This completes the proof.

The characterization result no longer holds for more than two players. In order to see this, consider the following three-player simultaneous move game, represented by its normal form.

\begin{tabular}{|l|l|l|l|}
\hline$g$ & $d$ & $e$ & $f$ \\
\hline$a$ & $3,3,0$ & $3,0,3$ & $0,2,0$ \\
\hline$b$ & $0,0,0$ & $0,0,0$ & $0,2,0$ \\
\hline$c$ & $2,0,0$ & $2,0,0$ & $2,2,0$ \\
\hline
\end{tabular}

\begin{tabular}{|l|l|l|l|}
\hline$h$ & $d$ & $e$ & $f$ \\
\hline$a$ & $0,0,0$ & $0,3,3$ & $0,2,0$ \\
\hline$b$ & $0,0,0$ & $3,3,0$ & $0,2,0$ \\
\hline$c$ & $2,0,0$ & $2,0,0$ & $2,2,0$ \\
\hline
\end{tabular}

\begin{tabular}{|l|l|l|l|}
\hline$i$ & $d$ & $e$ & $f$ \\
\hline$a$ & $0,0,2$ & $0,0,2$ & $0,2,2$ \\
\hline$b$ & $0,0,2$ & $0,0,2$ & $0,2,2$ \\
\hline$c$ & $2,0,2$ & $2,0,2$ & $2,2,2$ \\
\hline
\end{tabular}

Here, player 1 chooses between $a, b$ and $c$, player 2 chooses between $d, e$ and $f$, whereas player 3 chooses between $g, h$ and $i$. We show that $g$ is a minimally rationalizable strategy for player 3 , but not a Nash equilibrium strategy. Consider types $t_{1}, t_{2}, t_{3}$ such that $\mu_{1}\left(t_{1}, h_{0}\right)$ puts probability one on $\left(\left(e, t_{2}\right),\left(g, t_{3}\right)\right), \mu_{2}\left(t_{2}, h_{0}\right)$ puts probability one on $\left(\left(a, t_{1}\right),\left(h, t_{3}\right)\right)$ and $\mu_{3}\left(t_{3}, h_{0}\right)$ puts probability one on $\left(\left(a, t_{1}\right),\left(e, t_{2}\right)\right)$. Then, it may be verified that $t_{3}$ is persistently rationalizable for $(\mathcal{S}, u)$. Type $t_{3}$ has complexity 3 , which is the minimum possible complexity, and hence $t_{3}$ is minimally rationalizable. Since $g$ is sequentially rational for $t_{3}$, it follows that $g$ is minimally rationalizable.

Suppose that $g$ would be a Nash equilibrium strategy. Then, there would be a Nash equilibrium $\left(\mu_{1}, \mu_{2}, \mu_{3}\right)$ in first-order beliefs such that $g$ would be optimal against $\left(\mu_{1}, \mu_{2}\right)$. Here, 
$\mu_{i} \in \Delta\left(S_{i}\right)$ represents player $i$ 's opponents' common belief about player $i$ 's strategy choice. However, $g$ can only be optimal against $\left(\mu_{1}, \mu_{2}\right)$ if $\mu_{1}(a)>0$ and $\mu_{2}(e)>0$. Since $\left(\mu_{1}, \mu_{2}, \mu_{3}\right)$ is a Nash equilibrium, this implies that $a$ is optimal against $\left(\mu_{2}, \mu_{3}\right)$ and $e$ is optimal against $\left(\mu_{1}, \mu_{3}\right)$. This, in turn, implies that $\mu_{3}(g) \geq \frac{2}{3}$ and $\mu_{3}(h) \geq \frac{2}{3}$, which is clearly impossible. Hence, $g$ is not a Nash equilibrium strategy. The difference with minimal rationalizability is that the concept of Nash equilibrium requires player 3 to believe that players 1 and 2 hold the same belief about player 3's strategy choice, whereas minimal rationalizability does not impose such restriction. Indeed, types $t_{1}$ and $t_{2}$ above hold different beliefs about $t_{3}$ 's strategy choice, and this is what makes $g$ minimally rationalizable.

One direction of Theorem 6.1 remains true, however, if more than two players are allowed. Namely, in every $n$-player simultaneous move game, every Nash equilibrium strategy is minimally rationalizable. The proof is similar to the proof above and is left to the reader.

\section{Outside Option Games}

In this section, we shall prove that the concept of minimal rationalizability singles out the unique forward induction outcome in so-called outside option games as defined in van Damme (1989). An outside option game is a two-player game $(\mathcal{S}, u)$ with the following properties:

(1) At the beginning, player 1 may choose an outside option and leave the game or not choose the outside option and stay in the game; actions that will be denoted by Out and In, respectively.

(2) When taking the outside option, player 1 receives utility $u_{1}(O u t)$.

(3) If player 1 does not take the outside option, players 1 and 2 enter a simultaneous move game with action sets $A_{1}$ and $A_{2}$. In this subgame, there is a strict Nash equilibrium $\left(a_{1}^{*}, a_{2}^{*}\right)$ which yields player 1 utility $u_{1}\left(a_{1}^{*}, a_{2}^{*}\right)>u_{1}(O u t)$. All other Nash equilibria $\left(\mu_{1}, \mu_{2}\right)$ in first-order beliefs yield player 1 an expected utility strictly lower than $u_{1}($ Out $)$.

In van Damme (1989) it is argued that $\left(\operatorname{In}, a_{1}^{*}\right)$ and $a_{2}^{*}$ are the unique "forward induction strategies" in this game. The argument runs as follows. If player 2 observes that player 1 has not chosen the outside option, he should conclude that player 1 is heading for the only Nash equilibrium that dominates the outside option for him, that is, $\left(a_{1}^{*}, a_{2}^{*}\right)$. As such, he should believe that player 1 will play $a_{1}^{*}$, and hence player 2 should respond with $a_{2}^{*}$. Player 1 , anticipating on player 2 reasoning in this way, should therefore choose $\left(I n, a_{1}^{*}\right)$. In the following theorem, we prove that this argument is supported by the concept of minimal rationalizability.

Theorem 7.1. Let $(\mathcal{S}, u)$ be an outside option game in the sense of van Damme (1989). Then, the unique minimally rationalizable strategies for $(\mathcal{S}, u)$ are the forward induction strategies $\left(\operatorname{In}, a_{1}^{*}\right)$ and $a_{2}^{*}$.

Proof. Let $h_{1}$ and $h_{2}$ denote the information sets at which players 1 and 2 move in the simultaneous move game, respectively. We can construct types $t_{1}^{*}$ and $t_{2}^{*}$ with the following properties: (1) $t_{1}^{*}$ and $t_{2}^{*}$ have utility functions $u_{1}$ and $u_{2}$ throughout the game, where $u_{1}$ and $u_{2}$ are as specified by $(\mathcal{S}, u),(2) \mu_{1}\left(t_{1}^{*}, h_{0}\right)$ and $\mu_{1}\left(t_{1}^{*}, h_{1}\right)$ assign probability one to strategy $a_{2}^{*}$ and type $t_{2}^{*}$, and $(3) \mu_{2}\left(t_{2}^{*}, h_{0}\right)$ and $\mu_{2}\left(t_{2}^{*}, h_{2}\right)$ assign probability one to strategy $\left(\operatorname{In}, a_{1}^{*}\right)$ and type $t_{1}^{*}$. Then, it may be verified that $t_{1}^{*}$ and $t_{2}^{*}$ are persistently rationalizable for $(\mathcal{S}, u)$ with complexity 
2 , hence minimally rationalizable. Since $\left(\left(\operatorname{In}, a_{1}^{*}\right), t_{1}^{*}\right)$ and $\left(a_{2}^{*}, t_{2}^{*}\right)$ are sequentially rational, it follows that $\left(\operatorname{In}, a_{1}^{*}\right)$ and $a_{2}^{*}$ are minimally rationalizable.

Now, let $t_{1}$ be minimally rationalizable for $(\mathcal{S}, u)$. From the above, we must conclude that $t_{1}$ should have complexity 2 , that is, $T^{\infty}\left(t_{1}\right)=\left\{t_{1}, t_{2}\right\}$ for some $t_{2}$. Hence, $\mu_{1}\left(t_{1}, h_{0}\right)$ and $\mu_{1}\left(t_{1}, h_{1}\right)$ assign probability one to $t_{2}$, whereas $\mu_{2}\left(t_{2}, h_{0}\right)$ and $\mu_{2}\left(t_{2}, h_{2}\right)$ assign probability one to $t_{1}$. Since $t_{1}$ has utility function $u_{1}$ throughout the game, it follows, in particular, that $t_{2}$ believes at $h_{2}$ that player 1 has utility function $u_{1}$ at $h_{0}$ and $h_{1}$. Moreover, since $t_{2}$ has utility function $u_{2}$, it follows that $t_{1}$ believes at $h_{1}$ that player 2 has utility function $u_{2}$ at $h_{2}$. Let $\mu_{1}$ be the marginal of $\mu_{2}\left(t_{2}, h_{2}\right)$ on player 1's action set $A_{1}$ in the simultaneous move game, and let $\mu_{2}$ be the marginal of $\mu_{1}\left(t_{1}, h_{1}\right)$ on $A_{2}$. Since both $t_{1}$ and $t_{2}$ believe in sequential rationality, we may conclude that $\left(\mu_{1}, \mu_{2}\right)$ constitutes a Nash equilibrium in first-order beliefs in the subgame.

Since $t_{2}$ believes in sequential rationality, we know that $\mu_{2}\left(t_{2}, h_{2}\right)$ only assigns positive probability to player 1 strategies $\left(I n, a_{1}\right)$ that are optimal for $t_{1}$ at $h_{0}$. Hence, $\mu_{2}\left(t_{2}, h_{2}\right)\left(I n, a_{1}\right)>0$ only if $\left(I n, a_{1}\right)$ is optimal at $h_{0}$ given $\mu_{1}\left(t_{1}, h_{0}\right)$. Since $t_{1}$ satisfies updating consistency, we know that $\mu_{1}\left(t_{1}, h_{0}\right)=\mu_{1}\left(t_{1}, h_{1}\right)$. Recall that we have denoted the marginal of $\mu_{1}\left(t_{1}, h_{1}\right)$ on $A_{2}$ by $\mu_{2}$, and the marginal of $\mu_{2}\left(t_{2}, h_{2}\right)$ on $A_{1}$ by $\mu_{1}$. It thus follows that $\mu_{1}\left(a_{1}\right)>0$ only if $\left(\operatorname{In}, a_{1}\right)$ is optimal at $h_{0}$ given $\mu_{2}$. In particular, we have that $\mu_{1}\left(a_{1}\right)>0$ only if $u_{1}\left(a_{1}, \mu_{2}\right)>u_{1}($ Out $)$, where $u_{1}\left(a_{1}, \mu_{2}\right)$ denotes the expected utility of playing $a_{1}$ when having belief $\mu_{2}$ about player 2 's strategy choice.

In summary, we thus have that $\left(\mu_{1}, \mu_{2}\right)$ must constitute a Nash equilibrium, and $\mu_{1}\left(a_{1}\right)>0$ only if $u_{1}\left(a_{1}, \mu_{2}\right)>u_{1}(\mathrm{Out})$. However, by the definition of an outside option game, there is only one Nash equilibrium $\left(\mu_{1}, \mu_{2}\right)$ with this property, namely $\left(a_{1}^{*}, a_{2}^{*}\right)$. Hence, we may conclude that $\mu_{1}\left(t_{1}, h_{1}\right)$ puts probability one on $a_{2}^{*}$, and $\mu_{2}\left(t_{2}, h_{2}\right)$ puts probability one on $\left(\operatorname{In}, a_{1}^{*}\right)$. By updating consistency, we then have that $\mu_{1}\left(t_{1}, h_{0}\right)$ puts probability one on $a_{2}^{*}$. The unique strategy that is sequentially rational for $t_{1}$ is $\left(\operatorname{In}, a_{1}^{*}\right)$, and hence, $\left(\operatorname{In}, a_{1}^{*}\right)$ is the unique minimally rationalizable strategy for player 1 in $(\mathcal{S}, u)$.

Now, suppose that $t_{2}$ is minimally rationalizable for $(\mathcal{S}, u)$. Then, $t_{2}$ must have complexity 2 , which implies that $T^{\infty}\left(t_{2}\right)=\left\{t_{1}, t_{2}\right\}$ for some $t_{1}$. In particular, $\mu_{2}\left(t_{2}, h_{0}\right)$ and $\mu_{2}\left(t_{2}, h_{2}\right)$ must assign probability one to $t_{1}$. Since $t_{2}$ initially believes $u$, it follows that $u_{1}\left(t_{1}, h_{0}\right)=u_{1}$ and $u_{1}\left(t_{1}, h_{1}\right)=\left.u_{1}\right|_{Z\left(h_{1}\right)}$. Together with the observation that $t_{1}$ must be persistently rationalizable and must respect common belief about the event that types initially believe $u$, this implies that $t_{1}$ is persistently rationalizable for $(\mathcal{S}, u)$. Since $T^{\infty}\left(t_{2}\right)=\left\{t_{1}, t_{2}\right\}$, we have that $T^{\infty}\left(t_{1}\right)=\left\{t_{1}, t_{2}\right\}$ as well, and hence we may conclude that $t_{1}$ is minimally rationalizable for $(\mathcal{S}, u)$. Above we have already seen that in such a case, (In, $\left.a_{1}^{*}\right)$ is the unique strategy which is sequentially rational for $t_{1}$. Since $t_{2}$ believes in sequential rationality, and $\mu_{2}\left(t_{2}, h_{2}\right)$ assigns probability one to $t_{1}$, it follows that $\mu_{2}\left(t_{2}, h_{2}\right)$ must assign probability one to the strategy $\left(\operatorname{In}, a_{1}^{*}\right)$. But then, $a_{2}^{*}$ is the only strategy which is sequentially rational for $t_{2}$. Hence, $a_{2}^{*}$ is the only minimally rationalizable strategy for player 2 in $(\mathcal{S}, u)$. This completes the proof.

\section{Relation to Other Concepts}

The purpose of this section is to compare persistent and minimal rationalizability with the concepts of weak sequential rationalizability (Ben-Porath (1997)) and extensive form rational- 
izability (Pearce (1984) and Battigalli (1997)).

\subsection{Weak Sequential Rationalizability}

Formally speaking, the concept of weak sequential rationalizability as we use it, is an extension of the notion of common certainty of rationality at the beginning of the game, defined in BenPorath (1997) for the class of games with perfect information, to the general class of extensive form games. We first formally define weak sequential rationalizability within our epistemic framework. Let $\mathcal{S}$ be an extensive form structure and $u=\left(u_{i}\right)_{i \in I}$ a profile of utility functions. In the concept of weak sequential rationalizability, it is assumed that there be common belief about $u$ at every information set, and not only at the beginning of the game. By the latter, we formally mean that every type respects common belief about the event that all types $t_{i}$, at every information set $h_{i} \in H_{i}^{*}$, hold the utility function $u_{i}\left(t_{i}, h_{i}\right)=\left.u_{i}\right|_{Z\left(h_{i}\right)}$. Moreover, there is initial common belief about the event that types initially believe that players act sequentially rationally. In particular, a type is allowed to believe that an opponent is no longer acting sequentially rationally whenever this type finds out that the opponent has made a move that contradicts his initial beliefs. In order to state this property formally, we need the following definitions.

For a given type $t_{i}$ and opponent $j$, let

$$
T_{j}^{1}\left(t_{i}, h_{0}\right)=\operatorname{supp} \mu_{i}\left(t_{i}, h_{0} \mid T_{j}\right)
$$

be the set of player $j$ types that $t_{i}$ assigns positive probability to at the beginning of the game. Let $T_{i}^{1}\left(t_{i}, h_{0}\right)=\left\{t_{i}\right\}$, and let

$$
T^{1}\left(t_{i}, h_{0}\right)=\cup_{j \in I} T_{j}^{1}\left(t_{i}, h_{0}\right) .
$$

For all $k \geq 2$, let

$$
T^{k}\left(t_{i}, h_{0}\right)=\cup_{t \in T^{k-1}\left(t_{i}, h_{0}\right)} T^{1}\left(t, h_{0}\right) .
$$

Define $T^{\infty}\left(t_{i}, h_{0}\right)=\cup_{k \in \mathbb{N}} T^{k}\left(t_{i}, h_{0}\right)$. For a given event $\tilde{T} \subseteq \times_{j \in I} T_{j}$, we say that $t_{i}$ respects initial common belief about $\tilde{T}$ if $T^{\infty}\left(t_{i}, h_{0}\right) \subseteq \tilde{T}$.

For a given player $i$, recall that $\left(S_{-i} \times T_{-i}\right)^{s r}$ denotes the set of sequentially rational opponents' strategy-type pairs. Let $T_{i}^{s r}\left(h_{0}\right)$ be the set of player $i$ types $t_{i}$ that initially believe in sequential rationality, that is, with $\operatorname{supp} \mu_{i}\left(t_{i}, h_{0}\right) \subseteq\left(S_{-i} \times T_{-i}\right)^{s r}$.

Definition 8.1. A type $t_{i}$ is said to be weakly sequentially rationalizable for the game $(\mathcal{S}, u)$ if (1) $t_{i}$ holds utility function $u_{i}$, (2) respects common belief about $u$ at every information set, and (3) respects initial common belief about the event that types initially believe in sequential rationality. A strategy $s_{i}$ is called weakly sequentially rationalizable for $(\mathcal{S}, u)$ if there is a weakly sequentially rationalizable type $t_{i}$ for $(\mathcal{S}, u)$ such that $\left(s_{i}, t_{i}\right)$ is sequentially rational.

We shall now prove that for every extensive form game, persistently rationalizable strategies are always weakly sequentially rationalizable. The other direction is not true, since it is wellknown that weakly sequentially rationalizable strategies in a game with perfect information need not be backward induction strategies, while we have seen that persistent rationalizability always 
yields backward induction strategies in such games. One may even find examples of games with perfect information in which a profile of weakly sequentially rationalizable strategies need not lead to the backward induction outcome (see for instance Figure 1 in Ben-Porath (1997)).

Theorem 8.2. Let $\mathcal{S}$ be an extensive form structure and $u$ a profile of utility functions. Then, every persistently rationalizable strategy for $(\mathcal{S}, u)$ is weakly sequentially rationalizable for $(\mathcal{S}, u)$.

Proof. Let $s_{i}$ be persistently rationalizable for $(\mathcal{S}, u)$. Then, there is some type $t_{i}$ that is persistently rationalizable for $(\mathcal{S}, u)$ such that $\left(s_{i}, t_{i}\right)$ is sequentially rational. By definition, $t_{i}$ respects initial common belief about the event that types initially believe in sequential rationality. However, $t_{i}$ need not respect common belief about $u$ at every information set since $t_{i}$ may revise his belief about the opponents' utility functions as the game proceeds. However, the type $t_{i}$ may be transformed into a type $\tilde{t}_{i}$ such that (1) $\tilde{t}_{i}$ respects common belief about $u$ at all information sets, and (2) at every information set $h_{i}$, the marginal probability distribution of $\mu_{i}\left(\tilde{t}_{i}, h_{i}\right)$ on the opponents' strategies coincides with the corresponding marginal probability distribution of $\mu_{i}\left(t_{i}, h_{i}\right)$. Then, by construction, $\tilde{t}_{i}$ has utility function $u_{i}$, respects initial common belief about the event that types initially believe in sequential rationality, and respects common belief about $u$ at all information sets. Hence, $\tilde{t}_{i}$ is weakly sequentially rationalizable for $(\mathcal{S}, u)$. Moreover, since the beliefs of $\tilde{t}_{i}$ about the opponents' strategies coincide with $t_{i}$ 's beliefs at every information set $h_{i}$, and since $\left(s_{i}, t_{i}\right)$ is sequentially rational, it follows that $\left(s_{i}, \tilde{t}_{i}\right)$ is sequentially rational. We may thus conclude that $s_{i}$ is weakly sequentially rationalizable for $(\mathcal{S}, u)$, which completes the proof.

\subsection{Extensive Form Rationalizability}

The concept of extensive form rationalizability has been introduced in Pearce (1984) by means of an iterated elimination procedure. Later, Battigalli (1997) provided an alternative procedure that always leads to the same sets of strategies, whereas Battigalli and Siniscalchi (2002) give an epistemic foundation for the concept of extensive form rationalizability. Instead of delivering a precise definition of extensive form rationalizability, we shall restrict ourselves to a verbal expression of Battigalli and Siniscalchi's epistemic characterization in order to save space. This informal desciption will then be sufficient to prove that there is no general logical relationship between persistent rationalizability and extensive form rationalizability, at least in terms of strategy choices.

In the concept of extensive form rationalizability, the players' utility functions are never to be questioned during the game. Battigalli and Siniscalchi (2002) show that extensive form rationalizability can be characterized by the requirement that a player, at each of his information sets, should seek for the "highest possible degree of interactive belief in sequential rationality" that is still compatible with the event of reaching this information set, and should base his current and future beliefs upon this degree until it is contradicted at some later information set. As to illustrate the concept, consider the perfect information game in Figure 2, which is taken from Reny (1992b).

Suppose that player 2 observes that player 1 has chosen $r_{1}$ at his first move. Among the feasible strategies $\left(r_{1}, r_{3}\right)$ and $\left(r_{1}, d_{3}\right)$, only $\left(r_{1}, r_{3}\right)$ can possibly be a sequentially rational strategy for player 1 , given the restriction that player 2 should still believe at this point that player 


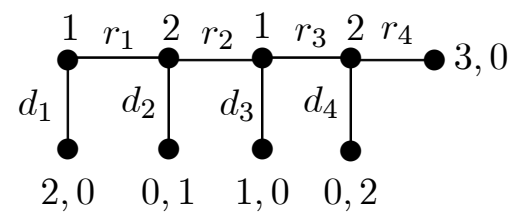

Figure 2

1's utility function is as depicted at the terminal nodes. As such, extensive form rationalizability requires player 2 to believe that player 1 has chosen $\left(r_{1}, r_{3}\right)$ after observing $r_{1}$. Given these beliefs, player 2 should choose $\left(r_{2}, d_{4}\right)$. If player 1 believes, at the beginning, that player 2 chooses $\left(r_{2}, d_{4}\right)$, player 1 should choose $d_{1}$. Hence, $d_{1}$ and $\left(r_{2}, d_{4}\right)$ are the unique extensive form rationalizable strategies in this game. Since the unique backward induction strategies are $d_{1}$ and $d_{2}$, we know by Theorem 5.1 that $d_{1}$ and $d_{2}$ are the unique persistently rationalizable (and hence unique minimally rationalizable) strategies in this game. We may thus conclude that a minimally rationalizable strategy need not be extensive form rationalizable, and an extensive form rationalizable strategy need not be persistently rationalizable.

In the perfect information game above, we see however that the concepts of extensive form rationalizability and persistent (minimal) rationalizability lead to the same unique outcome: the backward induction outcome $(2,0)$. This is a structural phenomenon for perfect information games, since Battigalli (1997) has shown that in a generic game with perfect information, every profile of extensive form rationalizable strategies leads to the backward induction outcome.

There are other games, however, where minimal rationalizability leads to outcomes that cannot be reached by extensive form rationalizability. Consider, for instance, the BurningMoney game in Figure 3, which is due to van Damme (1989) and Ben-Porath and Dekel (1992). In this game, player 1 may choose between burning a dollar (burn) or not burning a dollar (not) at round 1, after which players 1 and 2 face a simultaneous move game at round 2 . We show that $(n o t, e)$ and $(c, g)$ are the unique extensive from rationalizable strategies, leading to player 1's most preferred outcome $(3,1)$. Suppose that player 2 observes burn. Among the feasible strategies (burn, $a$ ) and (burn,,$b)$, only (burn, a) can possibly be sequentially rational, and hence extensive form rationalizability imposes that player 2 should believe that player 1 chooses (burn, a) after observing burn. As such, player 2 should choose $c$ after observing burn. Player 1, anticipating on player 2 choosing $c$ after burn, can thus guarantee 2 by choosing (burn, a). Player 2, realizing this, should thus believe that player 1 is choosing (not,e) after observing not, since (not, $f$ ) cannot give player 1 more than 2. Hence, player 2 should choose $g$ after not, which makes player 1 choosing $(n o t, e)$ at the beginning of the game. The only strategies that remain are thus (not,e) for player 1 and $(c, g)$ for player $2 .^{5}$

We will show, however, that minimal (and hence persistent) rationalizability may lead to

${ }^{5}$ Shimoji (2002) shows that in the more general class of Burning-Money games discussed in Ben-Porath and Dekel (1992), extensive form rationalizability always leads to the "forward induction outcome", that is, player 1's most preferred outcome in the game. 


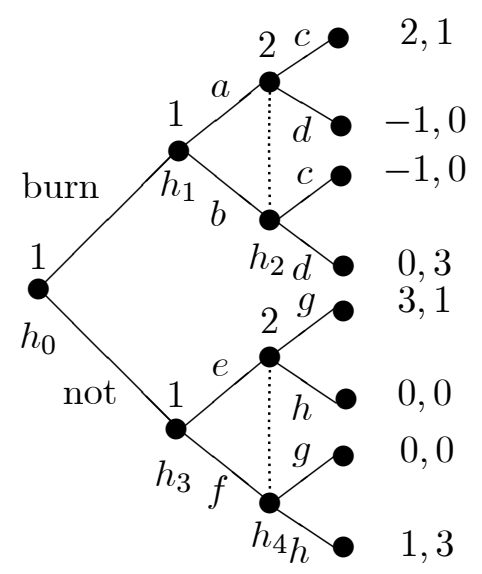

Figure 3

outcomes other than $(3,1)$. As a preparatory step, we first prove that any persistently rationalizable type in $(\mathcal{S}, u)$ should have a complexity strictly greater than 2 . Assume, on the contrary, that $t_{1}$ would be a persistently rationalizable type in $(\mathcal{S}, u)$ with complexity 2 . Then, there is some type $t_{2}$ such that $T^{\infty}\left(t_{1}\right)=\left\{t_{1}, t_{2}\right\}$, that is, $t_{1}$ believes at $h_{0}$ that player 2 has type $t_{2}$, and that $t_{2}$ believes at $h_{2}$ and $h_{4}$ that player 1 has type $t_{1}$. In particular, $t_{2}$ believes at $h_{2}$ and $h_{4}$ that player 1 has utility function $u_{1}$ as specified at the terminal nodes in Figure 3. Since $t_{2}$ should believe at $h_{2}$ that $t_{1}$ chooses sequentially rationally, $t_{2}$ should believe at $h_{2}$ that $t_{1}$ chooses (burn, $a$ ). Accordingly, $t_{1}$ should believe at $h_{0}$ that $t_{1}$ chooses $c$ after burn, and hence $t_{1}$ 's expected utility by choosing (burn, $a$ ) is 2 . Since $t_{2}$ should believe at $h_{4}$ that $t_{1}$ chooses sequentially rationally, $t_{2}$ should believe at $h_{4}$ that $t_{1}$ chooses (not,e). Hence, $t_{1}$ should believe that $t_{2}$ chooses $g$ after not, and hence $t_{1}$ 's expected utility by choosing (not,a) is 3 , which is greater than his expected utility by choosing (burn, $a$ ) or (burn, $b$ ). Therefore, $t_{2}$ cannot believe at $h_{2}$ that $t_{1}$ chooses sequentially rationally, and hence $t_{1}$ cannot be persistently rationalizable in $(\mathcal{S}, u)$ while having complexity 2 . Similarly, one may show that no persistently rationalizable type $t_{2}$ in $(\mathcal{S}, u)$ can have complexity 2 .

Now, let the utility functions $u_{1}$ and $u_{2}$ be as specified in Figure 3, and let $\tilde{u}_{1}$ be the utility function that coincides with $u_{1}$ at $Z\left(h_{3}\right)$, and for which $\tilde{u}_{1}(z)=u_{1}(z)-2$ for all $z \in Z\left(h_{1}\right)$. Let the types $t_{1}, \tilde{t}_{1}$ and $t_{2}$ be such that:

(1) $t_{1}$ has utility function $u_{1}$ and $\mu_{1}\left(t_{1}, h_{0}\right), \mu_{1}\left(t_{1}, h_{1}\right)$ and $\mu_{1}\left(t_{1}, h_{3}\right)$ assign probability one to $\left((c, h), t_{2}\right)$;

(2) $\tilde{t}_{1}$ has utility function $\tilde{u}_{1}$ and $\mu_{1}\left(t_{1}, h_{0}\right), \mu_{1}\left(t_{1}, h_{1}\right)$ and $\mu_{1}\left(t_{1}, h_{3}\right)$ assign probability one to $\left((c, h), t_{2}\right)$

(3) $t_{2}$ has utility function $u_{2}, \mu_{2}\left(t_{2}, h_{0}\right)$ and $\mu_{2}\left(t_{2}, h_{2}\right)$ assign probability one to ((burn, $\left.\left.a\right), t_{1}\right)$, and $\mu_{2}\left(t_{2}, h_{4}\right)$ assigns probability one to $\left((n o t, f), \tilde{t}_{1}\right)$.

It may be verified that $t_{1}$ and $t_{2}$ are persistently rationalizable for $(\mathcal{S}, u)$. Since both have complexity 3 , it follows that both $t_{1}$ and $t_{2}$ are minimally rationalizable for $(\mathcal{S}, u)$. The strategies 
(burn, $a)$ and $(c, h)$ are sequentially rational for $t_{1}$ and $t_{2}$, and hence (burn,a) and $(c, h)$ are minimally rationalizable for $(\mathcal{S}, u)$, leading to the outcome $(2,1)$. We may thus conclude that not every minimally rationalizable outcome is extensive form rationalizable in this game.

There are also games in which not every extensive form rationalizable outcome is minimally rationalizable. Consider the following two-player simultaneous move game (see Perea (2001), p.204) represented by its normal form.

\begin{tabular}{|l|l|l|l|}
\hline & $d$ & $e$ & $f$ \\
\hline$a$ & 3,3 & 0,0 & 3,2 \\
\hline$b$ & 0,0 & 3,3 & 3,2 \\
\hline$c$ & 2,0 & 2,0 & 2,2 \\
\hline
\end{tabular}

In this game $(\mathcal{S}, u)$, every strategy is rationalizable. Since extensive form rationalizability coincides with rationalizability in simultaneous move games, it follows that all strategies are extensive form rationalizable. However, strategy $c$ is not a Nash equilibrium strategy. Suppose, on the contrary, that $c$ were a Nash equilibrium strategy. Then, there should be some Nash equilibrium $\left(\mu_{1}, \mu_{2}\right) \in \Delta\left(S_{1}\right) \times \Delta\left(S_{2}\right)$ in first-order beliefs such that $c$ is a best response to $\mu_{2}$. This implies that $\mu_{2}(d)>0$ and $\mu_{2}(e)>0$. Hence, both $d$ and $e$ should be a best response to $\mu_{1}$, which is impossible. Consequently, $c$ cannot be a Nash equilibrium strategy. Since we know from Theorem 6.1 that the set of minimally rationalizable strategies coincides with the set of Nash equilibrium strategies in every two-player simultaneous move game, it follows that $c$ is not minimally rationalizable. In particular, the outcomes $(c, d),(c, e)$ and $(c, f)$ are extensive form rationalizable but not minimally rationalizable.

At this stage, it remains an open question whether in any given game, every extensive form rationalizable outcome is a persistently rationalizable outcome. Up to this point, I have not been able to provide a counterexample, nor to produce a general proof.

\section{References}

[1] Anscombe, F.J. and R. Aumann (1963), A definition of subjective probability, Annals of Mathematical Statistics 34, 199-205.

[2] Armbruster, W. and W. Böge (1979), Bayesian game theory, in: Game Theory and Related Topics (O. Moeschlin and D. Pallaschke, Eds.), North-Holland, Amsterdam.

[3] Asheim, G.B. (2000), On the epistemic foundation for backward induction, Memorandum No. 30, Department of Economics, University of Oslo.

[4] Asheim, G.B. (2001), Proper rationalizability in lexicographic beliefs, International Journal of Game Theory 30, 453-478.

[5] Asheim, G.B. and A. Perea (2002), Sequential and quasi-perfect rationalizability in extensive games, University of Oslo and Maastricht University.

[6] Aumann, R. (1995), Backward induction and common knowledge of rationality, Games and Economic Behavior 8, 6-19. 
[7] Aumann, R. and A. Brandenburger (1995), Epistemic conditions for Nash equilibrium, Econometrica 63, 1161-1180.

[8] Balkenborg, D. and E. Winter (1997), A necessary and sufficient epistemic condition for playing backward induction, Journal of Mathematical Economics 27, 325-345.

[9] Battigalli, P. (1996), Strategic independence and perfect Bayesian equilibria, Journal of Economic Theory 70, 201-234.

[10] Battigalli, P. (1997), On rationalizability in extensive games, Journal of Economic Theory 74, 40-61.

[11] Battigalli, P. (2003), Rationalizability in infinite, dynamic games with incomplete information, Research in Economics 57, 1-38.

[12] Battigalli, P. and M. Siniscalchi (2002), Strong belief and forward induction reasoning, Journal of Economic Theory 106, 356-391.

[13] Bernheim, B.D. (1984), Rationalizable strategic behavior, Econometrica 52, 1007-1028.

[14] Ben-Porath, E. (1997), Rationality, Nash equilibrium and backwards induction in perfectinformation games, Review of Economic Studies 64, 23-46.

[15] Ben-Porath, E. and E. Dekel (1992), Signaling future actions and the potential for sacrifice, Journal of Economic Theory 57, 36-51.

[16] Blume, L.E., Brandenburger, A. and E. Dekel (1991a), Lexicographic probabilities and choice under uncertainty, Econometrica 59, 61-79.

[17] Blume, L.E., Brandenburger, A. and E. Dekel (1991b), Lexicographic probabilities and equilibrium refinements, Econometrica 59, 81-98.

[18] Böge, W. and T.H. Eisele (1979), On solutions of bayesian games, International Journal of Game Theory 8, 193-215.

[19] Dekel, E. and D. Fudenberg (1990), Rational behavior and payoff uncertainty, Journal of Economic Theory 52, 243-267.

[20] Epstein, L. and T. Wang (1996), "Beliefs about beliefs" without probabilities, Econometrica 64, 1343-1373.

[21] Fudenberg, D., Kreps, D. and D. Levine (1988), On the robustness of equilibrium refinements, Journal of Economic Theory 44, 354-380.

[22] Harsanyi, J. (1973), Games with randomly disturbed payoffs: a new rationale for mixedstrategy equilibrium points, International Journal of Game Theory 2, 1-23.

[23] Mertens, J.-F. and S. Zamir (1985), Formulation of bayesian analysis for games with incomplete information, International Journal of Game Theory 14, 1-29. 
[24] Myerson, R.B. (1978), Refinements of the Nash equilibrium concept, International Journal of Game Theory 7, 73-80.

[25] Pearce, D. (1984), Rationalizable strategic behavior and the problem of perfection, Econometrica 52, 1029-1050.

[26] Perea, A. (2001), Rationality in Extensive Form Games, Kluwer Academic Publishers, Boston, Dordrecht.

[27] Perea, A. (2002), Forward induction and the minimum revision principle, Meteor Research Memorandum RM/02/010, Maastricht University.

[28] Perea, A. (2003), Proper rationalizability and belief revision in dynamic games, Maastricht University.

[29] Reny, P.J. (1992a), Rationality in extensive-form games, Journal of Economic Perspectives 6, $103-118$.

[30] Reny, P.J. (1992b), Backward induction, normal form perfection and explicable equilibria, Econometrica 60, 627-649.

[31] Reny, P.J. (1993), Common belief and the theory of games with perfect information, Journal of Economic Theory 59, 257-274.

[32] Rubinstein, A. (1991), Comments on the interpretation of game theory, Econometrica 59, 909-924.

[33] Samet, D. (1996), Hypothetical knowledge and games with perfect information, Games and Economic Behavior 17, 230-251.

[34] Savage, L.J. (1954), The Foundations of Statistics, Wiley, New York.

[35] Schuhmacher, F. (1999), Proper rationalizability and backward induction, International Journal of Game Theory 28, 599-615.

[36] Shimoji, M. (2002), On forward induction in money-burning games, Economic Theory 19, 637-648.

[37] Stalnaker, R. (1998), Belief revision in games: forward and backward induction, Mathematical Social Sciences 36, 31-56.

[38] Stinchcombe, M.B. and K.G. Zauner (2002), Inference preserving perturbations of extensive-form games: the agent normal form approach, Mimeo.

[39] Tan, T. and S.R.C. Werlang (1988), The bayesian foundations of solution concepts of games, Journal of Economic Theory 45, 370-391.

[40] van Damme, E. (1989), Stable equilibria and forward induction, Journal of Economic Theory 48, 476-496. 
[41] Zauner, K.G. (2002), The existence of equilibrium in games with randomly perturbed payoffs and applications to experimental economics, Mathematical Social Sciences 44, 115-120. 